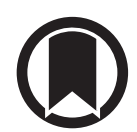

CrossMark

\title{
Young and middle-aged adults with airflow limitation according to lower limit of normal but not fixed ratio have high morbidity and poor survival: a population-based prospective cohort study
}

\author{
Yunus C̦olak ${ }^{1,2}$, Shoaib Afzal ${ }^{1,2}$, Børge G. Nordestgaard ${ }^{1,2,3}$, Jørgen Vestbo ${ }^{4}$ and \\ Peter Lange $\mathrm{e}^{2,3,5,6}$
}

Affiliations: ${ }^{1}$ Dept of Clinical Biochemistry, Herlev and Gentofte Hospital, Copenhagen University Hospital, Herlev, Denmark. ${ }^{2}$ The Copenhagen General Population Study, Herlev and Gentofte Hospital, Copenhagen University Hospital, Herlev, Denmark. ${ }^{3}$ Faculty of Health and Medical Sciences, University of Copenhagen, Copenhagen, Denmark. "Division of Infection, Immunity and Respiratory Medicine, School of Biological Sciences, Manchester Academic Health Science Centre, University of Manchester, and Manchester University NHS Foundation Trust, Manchester, UK. ${ }^{5}$ Dept of Public Health, Section of Social Medicine, University of Copenhagen, Copenhagen, Denmark. 'Dept of Internal Medicine, Section of Respiratory Medicine, Herlev and Gentofte Hospital, Copenhagen University Hospital, Herlev, Denmark.

Correspondence: P. Lange, Dept of Public Health, Section of Social Medicine, University of Copenhagen, Øster Farimagsgade 5, Postal Box 2099, DK-1015 Copenhagen K, Denmark. E-mail: Peter.Langedsund.ku.dk

@ERSpublications

Young and middle-aged adults with airflow limitation according to LLN but not fixed ratio have poor prognosis http://ow.ly/QwOP30iiU11

Cite this article as: Çolak Y, Afzal S, Nordestgaard BG, et al. Young and middle-aged adults with airflow limitation according to lower limit of normal but not fixed ratio have high morbidity and poor survival: a population-based prospective cohort study. Eur Respir J 2018; 51: 1702681 [https://doi.org/10.1183/ 13993003.02681-2017].

ABSTRACT A presumed consequence of using a fixed ratio for the definition of airflow limitation (AFL) has been overdiagnosis among older individuals and underdiagnosis among younger individuals. However, the prognosis of younger individuals with potentially underdiagnosed AFL is poorly described. We hypothesised that potential underdiagnosis of AFL at a younger age is associated with poor prognosis.

We assigned 95288 participants aged 20-100 years from the Copenhagen General Population Study into the following groups: individuals without AFL with forced expiratory volume in $1 \mathrm{~s}$ (FEV1)/forced vital capacity (FVC) $\geqslant 0.70$ and $\geqslant$ lower limit of normal (LLN) ( $\mathrm{n}=78779,83 \%$ ); individuals with potentially underdiagnosed AFL with FEV1/FVC $\geqslant 0.70$ and <LLN ( $\mathrm{n}=1056,1 \%)$; individuals with potentially overdiagnosed AFL with FEV1/FVC $<0.70$ and $\geqslant \operatorname{LLN}(\mathrm{n}=3088,3 \%)$; and individuals with AFL with FEV1/ FVC $<0.70$ and $<\operatorname{LLN}(\mathrm{n}=12365,13 \%)$. We assessed risk of exacerbations, pneumonias, ischaemic heart disease, heart failure and all-cause mortality. Median follow-up was 6.0 years (range: 2 days-11 years).

Compared to individuals without AFL, individuals with potentially underdiagnosed AFL had an increased risk of morbidity and mortality with age- and sex-adjusted hazard ratios (HR) of 2.7 (95\% CI: 1.7-4.5) for pneumonias, 2.3 (95\% CI: 1.2-4.5) for heart failure, and 3.1 (95\% CI: 2.1-4.6) for all-cause mortality.

Young and middle-aged adults with AFL according to LLN but not fixed ratio experience increased respiratory and cardiovascular morbidity and early death.

This article has supplementary material available from erj.ersjournals.com

Received: Dec 222017 | Accepted after revision: Feb 042018

Copyright @ERS 2018 


\section{Introduction}

Underdiagnosis of chronic obstructive pulmonary disease (COPD) is substantial and associated with poor prognosis, even among asymptomatic individuals [1]. However, the presence of airflow limitation (AFL) with a low ratio of forced expiratory volume in $1 \mathrm{~s}$ (FEV1) and forced vital capacity (FVC) can be easily determined using spirometry $[2,3]$. Although the use of spirometry is not recommended for the screening of asymptomatic adults, it is encouraged in order to allow clinicians to pursue active case-finding in high-risk populations and to detect COPD early before severe AFL develops [4]. The appropriate definition of AFL is hotly debated. On the one hand, the use of a fixed ratio (i.e. FEV $1 / \mathrm{FVC}<0.70$ ) is a simple approach in a busy daily clinical setting [2,3], while, on the other hand, the fixed ratio does not account for the normal age-dependent decline in lung function, which leads to a lower ratio of $\mathrm{FEV}_{1} / \mathrm{FVC}$ with increasing age [5]. Thus, a presumed consequence of using a fixed ratio would be overdiagnosis among older individuals and underdiagnosis among younger individuals [5,6]. The latter situation is especially worrisome, as it may hamper smoking cessation advice and initiation of early treatment. Instead of the fixed ratio, it has been recommended to use the lower 5th percentile of the predicted value for FEV1/FVC (i.e. FEV1/FVC <lower limit of normal (LLN)), as such an approach will not be prone to an age-dependent variation in lung function and will also be able to account for other biological differences such as gender and race [7, 8]. Previous studies have mainly focused on the prognosis of older individuals with potentially overdiagnosed AFL ( FEV $/$ FVC $<0.70$ and $\geqslant L L N)$. However, the prognosis of younger individuals with potentially underdiagnosed AFL (FEV $1 / \mathrm{FVC} \geqslant 0.70$ and $<\mathrm{LLN})$ is poorly described.

In the present study, we investigated the prognosis of potentially underdiagnosed AFL in younger individuals from the general population, as defined by the LLN but not the fixed ratio. For this purpose, we used the Copenhagen General Population Study including 95288 randomly selected individuals aged 20-100 years. We hypothesised that potential underdiagnosis of AFL at a younger age is associated with poor prognosis.

\section{Methods}

\section{Study design and participants}

We recruited 95288 individuals aged 20-100 years from the Copenhagen General Population Study, a Danish population-based prospective cohort study initiated in November 26, 2003 with ongoing enrolment [1]. In the present study, we had information until July 10, 2013. In Denmark, all individuals are assigned a unique identification number at birth or immigration and recorded in the National Danish Civil Registration System. Based on age and sex, individuals living in the Capital Region of Denmark were randomly selected from this registration system using the unique identification number to reflect the adult white Danish population of Danish descent. Response rate was approximately $45 \%$ and all participants completed a comprehensive questionnaire, underwent a physical health examination and provided blood for biochemical analysis. Questionnaires were reviewed on the day of attendance by a healthcare professional together with the participant. The study was approved by Herlev and Gentofte Hospital and the regional ethics committee (H-KF-01-144/01) and conducted according to the Declaration of Helsinki. All participants provided written informed consent.

\section{Formation of the clinical groups}

Lung function was determined using spirometry with prebronchodilator measurements of FEV1 and FVC. Spirometry use in the Copenhagen General Population Study has undergone a rigorous validation process [9]. Predicted values were calculated separately for men and women using internally derived reference values based on a subsample of 11288 healthy, asymptomatic, never-smoking individuals, with age and height as covariates [9]. A detailed description of spirometry procedures and the questions used to assess characteristics can be found in the supplementary material. The LLN, defined as the lower 5th percentile of the predicted values for FEV1/FVC, was calculated as the mean value minus 1.645 standard deviations. Individuals were assigned to one of four exclusive subgroups according to different criteria for AFL (figure 1), as follows: 1) No AFL (individuals with FEV1/FVC $\geqslant 0.70$ and $\geqslant L L N$ ); 2) Potentially underdiagnosed AFL (individuals with $\mathrm{FEV} 1 / \mathrm{FVC} \geqslant 0.70$ but $<\mathrm{LLN}$ ); 3) Potentially overdiagnosed AFL (individuals with FEV1/FVC $<0.70$ but $\geqslant L L N) ; 4)$ AFL (individuals with FEV1/FVC $<0.70$ and $<$ LLN).

\section{Outcomes}

Severe exacerbations of obstructive lung disease, both COPD and asthma-related (International Classification of Diseases (ICD) codes ICD-8: 491-493 and ICD-10: J41-J46), and pneumonias (ICD-8: 480-486 and ICD-10: J12-J18), were defined as acute emergency department visits or hospital admissions with the mentioned primary diagnosis. Ischaemic heart disease (ICD-8: 410-414 and ICD-10: I20-I25) and heart failure (ICD-8: 427.09-427.11 and ICD-10: I50) were defined as inpatient and outpatient hospital contacts with the mentioned primary or secondary diagnosis. Information was obtained from the National 

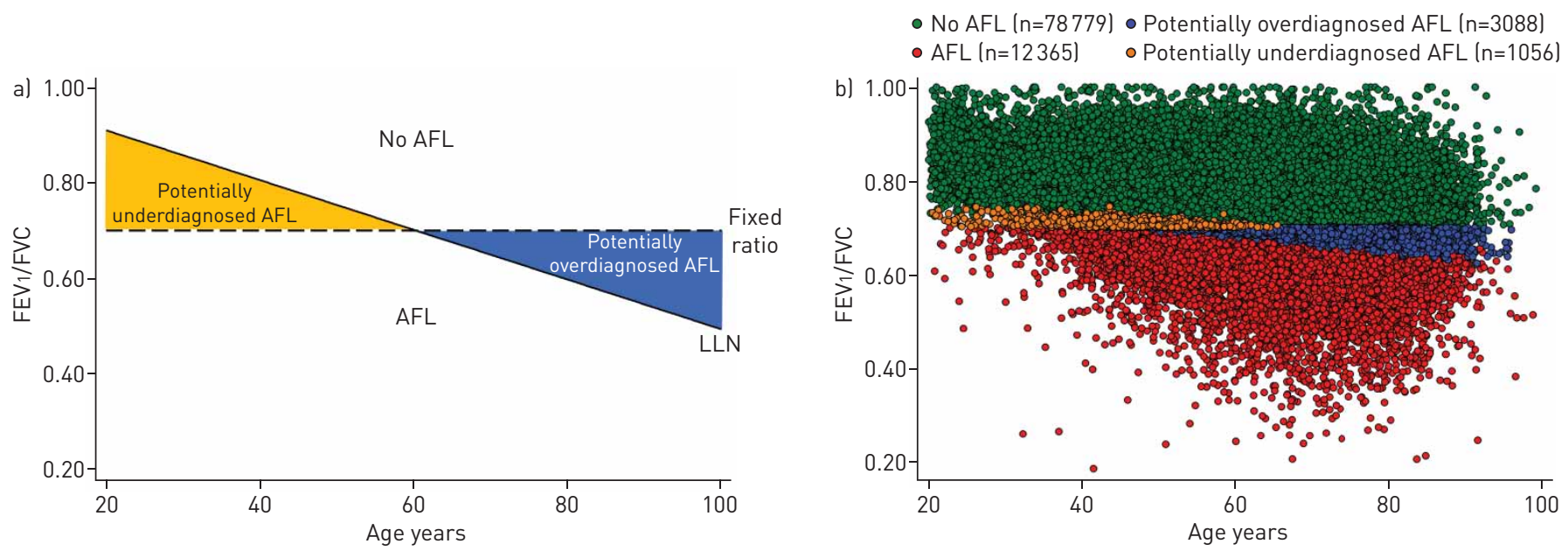

FIGURE 1 Definition of airflow limitation (AFL) and differences between the lower limit of normal and the fixed ratio criteria. (a) A theoretical depiction based on two different criteria for AFL, the fixed ratio and the lower limit of normal (LLN). (b) Assignment of individuals from the Copenhagen General Population Study to different criteria for AFL. No AFL was defined as forced expiratory volume in $1 \mathrm{~s}$ (FEV1)/forced vital capacity $(F V C) \geqslant 0.70$ and FEV $1 / F V C \geqslant L L N$. Potentially underdiagnosed AFL was defined as FEV $1 / F V C \geqslant 0.70$ and FEV $1 / F V C<L L N$. Potentially overdiagnosed AFL was defined as FEV $1 / F V C<0.70$ and $F E V_{1} / F V C \geqslant L L N$. AFL was defined as FEV $1 / F V C<0.70$ and FEV $1 / F V C<L L N$.

Danish Patient Registry, which covers all public and private Danish hospitals, recorded until November 10, 2014. Denmark used Revision 8 of the ICD (ICD-8) until January 01, 1994 and then proceeded directly to Revision 10 (ICD-10) after this date. Information on vital status was obtained from the National Danish Civil Registration System, recorded until November 14, 2014. As follow-up was performed using the register (with a linkage based on the unique identification number provided to everyone at birth or immigration), no person was lost to follow-up and individuals who emigrated were censored at the date of emigration $(n=376)$. All diagnoses recorded in the registries were made by a medical doctor according to national law.

\section{Statistical analyses}

Wilcoxon rank-sum and Pearson Chi-squared tests were used for comparisons. Since age differed substantially between the clinical groups, logistic- and linear-regression models were used to account for this in the clinical attributes; however, unadjusted differences are also provided. Kernel density estimation was used to illustrate the age distribution within the clinical groups and Cox proportional hazard models were used to determine the prognosis. For exacerbations and pneumonias, multiple failure-time analysis was carried out using the Andersen-Gill approach (meaning that individuals were at risk of recurrent events); otherwise, a single failure-time analysis approach was used. Analyses were used with left truncation and age as the underlying timescale, and, due to the findings with regard to pneumonia and heart failure, competing risk analysis were also carried out using the methods proposed by Fine and Gray (the competing event being all-cause mortality). Since an approach using multiple failure-time analysis does not work using Fine and Gray, an approach using single failure-time analysis was used instead. Kaplan-Meier analysis was used for all-cause mortality and area-proportional Venn diagrams were also created [10]. In the sensitivity analyses, we included other outcome related potential confounders, stratified according to the presence of relevant symptomatology (i.e. chronic mucus hypersecretion, dyspnoea, wheezing and/or cough) and restricted the analysis to individuals without AFL according to quartiles of FEV1/FVC. A detailed description of the questions used to assess potential confounders and symptoms can be found in the supplementary material. All analyses were performed using STATA/SE 13.1 for Windows (StataCorp, College Station, Texas, US) and a two-sided p-value $<0.05$ was considered as significant.

\section{Results}

Among the 95288 participants, 78779 individuals (83\%) did not have AFL, 1056 individuals (1\%) had potentially underdiagnosed AFL, 3088 individuals (3\%) had potentially overdiagnosed AFL and 12365 individuals (13\%) had AFL. Among individuals with potentially underdiagnosed AFL, 76\% were aged 2050 years, whereas this fraction was $33 \%$ among individuals without AFL (figure 2). Individuals with potentially underdiagnosed AFL constituted $3 \%$ of younger individuals aged $20-50$ years and $<0.5 \%$ of older individuals aged 50-100 years. Median follow-up was 6.0 years (range: 2 days to 11 years) and, 


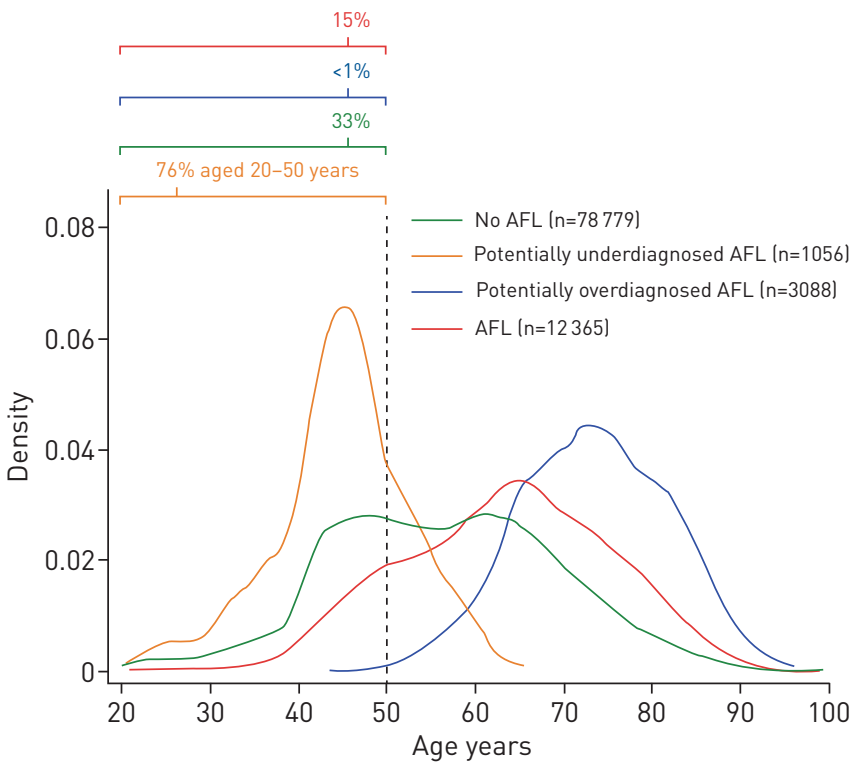

FIGURE 2 Age distribution within clinical groups of airflow limitation (AFL) illustrated using Kernel density estimation. No AFL was defined as forced expiratory volume in $1 \mathrm{~s}(F E V 1) /$ forced vital capacity $(F V C) \geqslant 0.70$ and FEV $1 / F V C \geqslant$ lower limit of normal (LLN). Potentially underdiagnosed AFL was defined as FEV $1 / F V C \geqslant 0.70$ and FEV $1 / F V C<L L N$. Potentially overdiagnosed $A F L$ was defined as $F E V_{1} / F V C<0.70$ and $F E V_{1} / F V C \geqslant L L N$. AFL was defined as $\mathrm{FEV}_{1} / \mathrm{FVC}<0.70$ and $\mathrm{FEV} 1 / \mathrm{FVC}<\mathrm{LLN}$.

during this period, we observed 2073 severe exacerbations of obstructive lung disease, 4487 pneumonias, 3859 ischaemic heart disease events, 2046 heart failures and 5260 deaths. Since the main purpose was to investigate individuals with potentially underdiagnosed AFL, the analyses including individuals with potentially overdiagnosed AFL and individuals with AFL are given in the supplementary material.

Compared to individuals without AFL, individuals with potentially underdiagnosed AFL were younger with a median age of 45 years versus 56 years and were more often current smokers with a prevalence of $28 \%$ versus $15 \%$ (table 1); however, the difference in cumulative tobacco consumption was small (16 pack-years versus 14 pack-years). After taking age and sex into account and compared to individuals without AFL, individuals with potentially underdiagnosed AFL had a higher prevalence of asthma (10\%

TABLE 1 Baseline characteristics of 95288 individuals according to clinical groups of airflow limitation (AFL) in the Copenhagen General Population Study

\begin{tabular}{|c|c|c|c|c|c|c|c|c|}
\hline & \multirow{2}{*}{$\frac{\text { No AFL }}{(n=78779)}$} & \multirow{2}{*}{$\frac{A F L}{(n=12365)}$} & \multicolumn{3}{|c|}{ Potentially underdiagnosed AFL } & \multicolumn{3}{|c|}{ Potentially overdiagnosed AFL } \\
\hline & & & $(n=1056)$ & $\begin{array}{l}\text { p versus } \\
\text { No AFL }\end{array}$ & $\begin{array}{c}\text { p versus } \\
\text { AFL }\end{array}$ & $(n=3088)$ & $\begin{array}{l}\text { p versus } \\
\text { No AFL }\end{array}$ & $\begin{array}{c}\text { p versus } \\
\text { AFL }\end{array}$ \\
\hline Male & $35037(44)$ & $6182(50)$ & $434(41)$ & 0.03 & $<0.0001$ & $1230(40)$ & $<0.0001$ & $<0.0001$ \\
\hline FEV $1 \%$ predicted & $98(89-108)$ & $80(66-91)$ & 90 (82-98) & $<0.0001$ & $<0.0001$ & $91(80-102)$ & $<0.0001$ & $<0.0001$ \\
\hline FVC $\%$ predicted & $99(90-108)$ & $99(85-11)$ & $104(94-112)$ & $<0.0001$ & $<0.0001$ & $101(88-113)$ & $<0.0001$ & $<0.0001$ \\
\hline Never smokers & $36152(46)$ & $2988(24)$ & 416 (39) & $<0.0001$ & $<0.0001$ & 1034 (33) & $<0.0001$ & $<0.0001$ \\
\hline Former smokers & 30584 (39) & $5500(44)$ & 345 (33) & $<0.0001$ & $<0.0001$ & 1518 (49) & $<0.0001$ & $<0.0001$ \\
\hline Current smokers & $12007(15)$ & $3863(31)$ & $294(28)$ & $<0.0001$ & 0.02 & $533(17)$ & 0.002 & $<0.0001$ \\
\hline \multicolumn{9}{|c|}{ Cumulative tobacco consumption ${ }^{\#}$} \\
\hline Consumption pack-years & $14(5-27)$ & $27(14-42)$ & $16(6-28)$ & 0.04 & $<0.0001$ & $21(9-37)$ & $<0.0001$ & $<0.0001$ \\
\hline
\end{tabular}

Data are presented as $\mathrm{n}(\%)$ or median (25th-75th percentile). p-Values were obtained from Wilcoxon rank-sum or Pearson Chi-squared tests. No airflow limitation (AFL) was defined as forced expiratory volume in $1 \mathrm{~s}(\mathrm{FEV} 1) /$ forced vital capacity $(\mathrm{FVC}) \geqslant 0.70$ and $F E V 1 / F V C \geqslant l o w e r$ limit of normal (LLN). Potentially underdiagnosed AFL was defined as FEV $1 / F V C \geqslant 0.70$ and FEV $1 / F V C<L L N$. Potentially overdiagnosed $A F L$ was defined as $\mathrm{FEV}_{1} / \mathrm{FVC}<0.70$ and $\mathrm{FEV}_{1} / \mathrm{FVC} \geqslant \mathrm{LLN}$. AFL was defined as $\mathrm{FEV}_{1} / \mathrm{FVC}<0.70$ and $\mathrm{FEV}_{1} / \mathrm{FVC}<\mathrm{LLN}$. * : Consumption was only calculated for former and current smokers. 
TABLE 2 Age- and sex-adjusted comorbidities, severity of disease and healthcare use among 95288 individuals according to clinical groups of airflow limitation (AFL) in the Copenhagen General Population Study

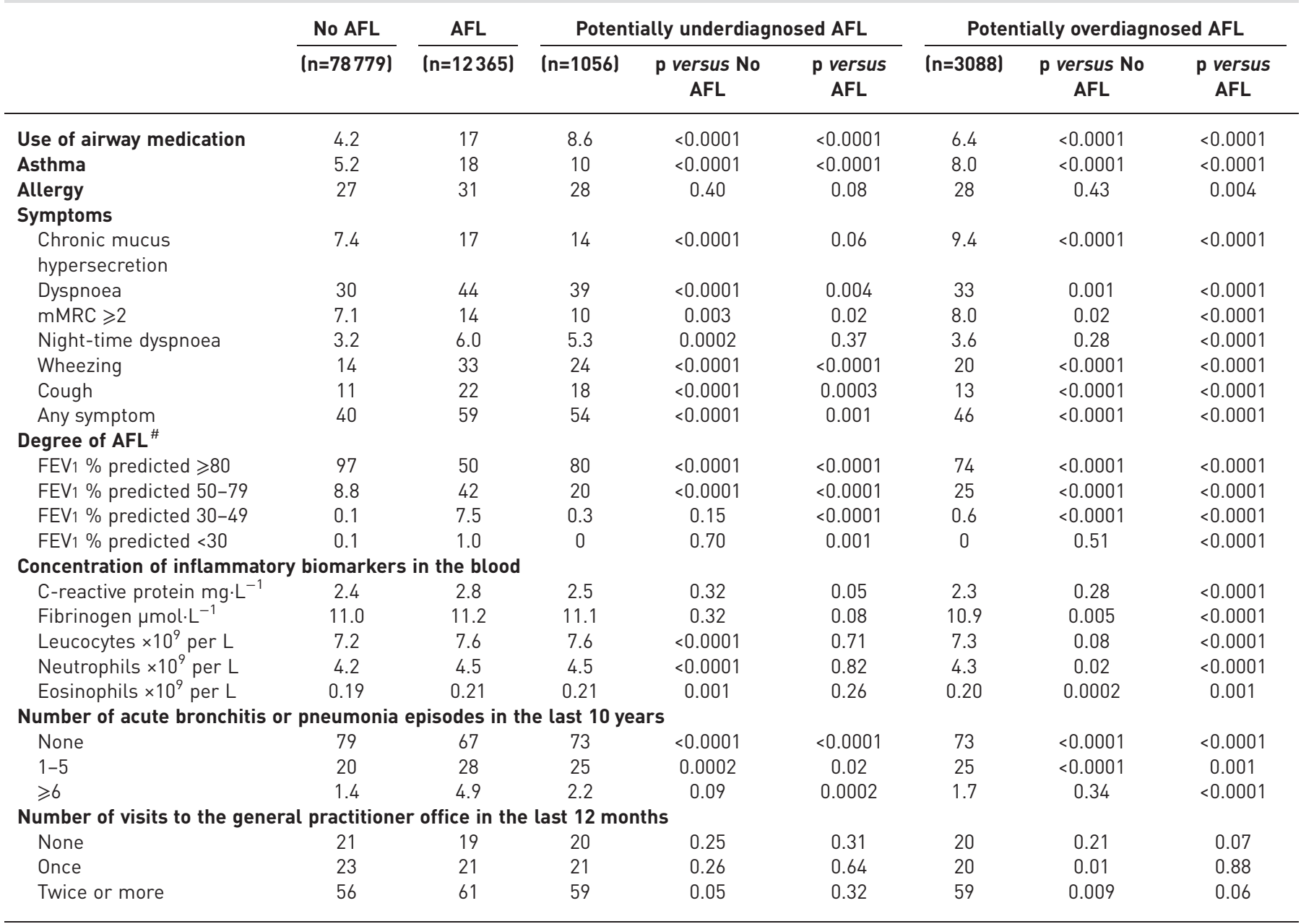

Data are presented as \% and means. Percentages are age- and sex-adjusted, obtained from logistic regression models. Mean concentrations of inflammatory biomarkers in the blood are age- and sex-adjusted, obtained from linear regression models. p-Values are obtained from Wald tests. No airflow limitation (AFL) was defined as forced expiratory volume in $1 \mathrm{~s}(\mathrm{FEV} 1) /$ forced vital capacity $(\mathrm{FVC}) \geqslant 0.70$ and $\mathrm{FEV} 1 / \mathrm{FVC} \geqslant \mathrm{lower}$ limit of normal (LLN). Potentially underdiagnosed AFL was defined as FEV1/FVC $\geqslant 0.70$ and FEV $1 / F V C<L L N$. Potentially overdiagnosed AFL was defined as $F E V_{1} / F V C<0.70$ and $F E V_{1} / F V C \geqslant L L N$. AFL was defined as FEV $1 / F V C<0.70$ and FEV $1 / F V C<L L N$. GP: general practitioner; mMRC: modified Medical Research Council dyspnoea scale. \#: Since FEV $1 \%$ predicted is already age- and sex-adjusted, no further adjustment was performed. p-Values are obtained from Pearson Chi-squared tests.

versus $5.2 \%)$, respiratory symptoms (54\% versus $40 \%)$ and airway medication use $(8.6 \%$ versus $4.2 \%)$ (table 2). Furthermore, $80 \%$ had a mild form of AFL with $\%$ predicted FEV $1 \geqslant 80 \%$. Only minor differences could be observed in concentrations of inflammatory biomarkers in the blood and in previous utilisation of healthcare compared to individuals without AFL. Results were similar without adjustment (see supplementary table S1).

Individuals with potentially underdiagnosed AFL had an increased risk of pneumonia, heart failure and all-cause mortality but not of exacerbations of obstructive lung disease or ischaemic heart disease compared to individuals without AFL (figure 3 and supplementary figure S1). Age- and sex-adjusted hazard ratios (HRs) were 2.7 (95\% CI 1.7-4.5) for pneumonias, 2.3 (95\% CI 1.2-4.5) for heart failure and 3.1 (95\% CI 2.1-4.6) for all-cause mortality (figure 3, left panel). After additional adjustment for smoking status and cumulative tobacco consumption, the risk estimates were similar (figure 3, right panel). The increased risk of pneumonia and heart failure persisted in a competing risk analysis with the competing event being all-cause mortality (figure 4). Only minor overlaps could be observed among individuals with potentially underdiagnosed AFL experiencing pneumonia, heart failure and/or death (figure 5). In sensitivity analyses, after additional adjustment for other potential confounders, risk estimates were similar 
Adjusted for age and sex

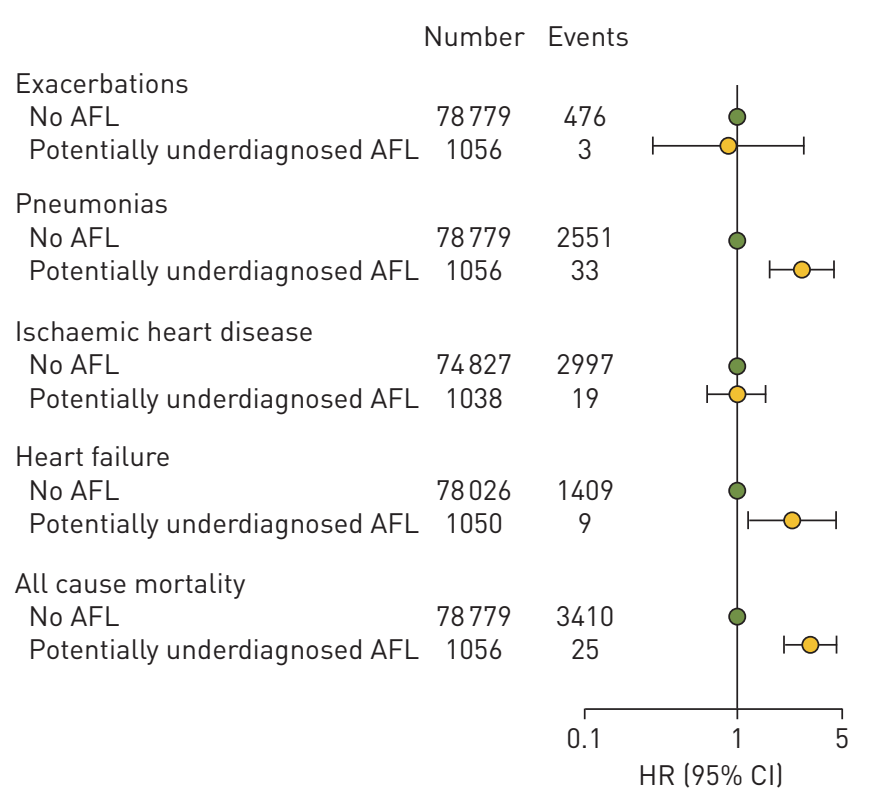

$\operatorname{HR}(95 \% \mathrm{Cl}) \quad \mathrm{p}$-value

Additionally adjusted for smoking status and cumulative tobacco consumption

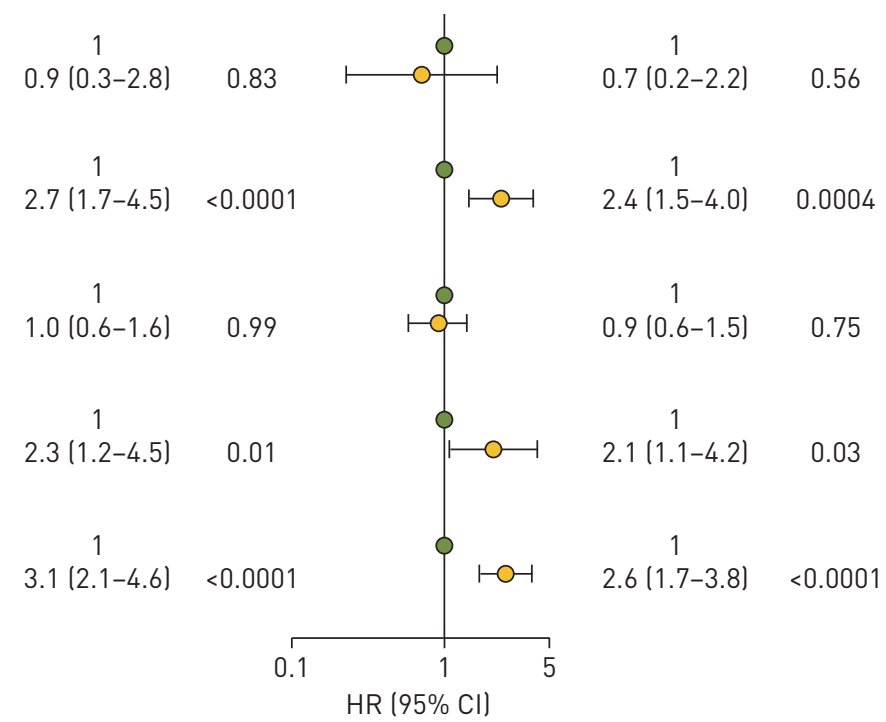

FIGURE 3 Prognosis of individuals with potentially underdiagnosed airflow limitation (AFL). Risk of exacerbations and pneumonias was assessed using multiple failure-time analysis; otherwise, a single failure-time analysis was used. p-Values were obtained from Wald tests. No AFL was defined as forced expiratory volume in $1 \mathrm{~s}$ (FEV1)/forced vital capacity (FVC) $\geqslant 0.70$ and FEV $1 / F V C \geqslant$ lower limit of normal (LLN). Potentially underdiagnosed $\mathrm{AFL}$ was defined as $\mathrm{FEV} 1 / \mathrm{FVC} \geqslant 0.70$ and $\mathrm{FEV} 1 / \mathrm{FVC}<\mathrm{LLN}$. Cl: confidence interval; HR: hazard ratio.

(see supplementary tables S2 and S3 and supplementary figure S1, right panel). Furthermore, after stratification according to presence of respiratory symptoms, risk estimates were slightly attenuated for asymptomatic individuals and were higher for symptomatic individuals (see supplementary figure S2). Lastly, among individuals without AFL, those in the 1st quartile of FEV1/FVC (range: 0.70-0.76) had an increased risk of exacerbations of obstructive lung disease and all-cause mortality, although not of pneumonias, ischaemic heart disease or heart failure, compared to those in the 4th quartile of FEV1/FVC (range: 0.83-1.00) (see supplementary figure S3). This suggests that even within the range of normal lung function, there seems to be an association of lower lung function with poor prognosis.

Individuals with potentially overdiagnosed AFL had an increased risk of exacerbations and pneumonias but not of ischaemic heart disease, heart failure or all-cause mortality compared to individuals without AFL, despite adjustment for potential confounders (see supplementary figure S1). Age- and sex-adjusted HRs were 2.6 (95\% CI 1.8-3.8) for exacerbations and 1.5 (95\% CI 1.3-1.8) for pneumonias (see supplementary figure S1, left panel). After stratification according to the presence of respiratory symptoms, risk estimates were slightly attenuated for asymptomatic individuals and higher for symptomatic individuals (see supplementary figure S2). In addition, symptomatic individuals also had an increased risk of ischaemic heart disease, heart failure and all-cause mortality.

\section{Discussion}

In a large sample from the general population, we found that younger individuals with AFL defined using the LLN criterion but not the fixed ratio criterion had a 2.7 -fold risk of pneumonias, a 2.3-fold risk of heart failure and a 3.1-fold risk of all-cause mortality.

Only $12 \%$ of individuals with potentially underdiagnosed AFL were reported to have asthma. During follow-up, these individuals did not have an increased risk of COPD or asthma-related exacerbations compared to individuals without AFL. A likely explanation is that these non-asthmatic individuals may have underdeveloped lungs. Normal lung development is characterised by a rapid increase in lung function during childhood and peaks in adulthood around 20-25 years of age. This is then replaced by a plateau phase, with preservation of maximally attained lung function for approximately 10 years, before a steady decline with increasing age. Therefore, AFL among these young individuals may be due to an insufficient peak of lung function in adulthood [11-14]. Underdeveloped lungs in young adulthood with low maximally attained lung function is a risk factor for respiratory disease when older and can even, through normal lung function decline, lead to the development of COPD later in life [15]. 

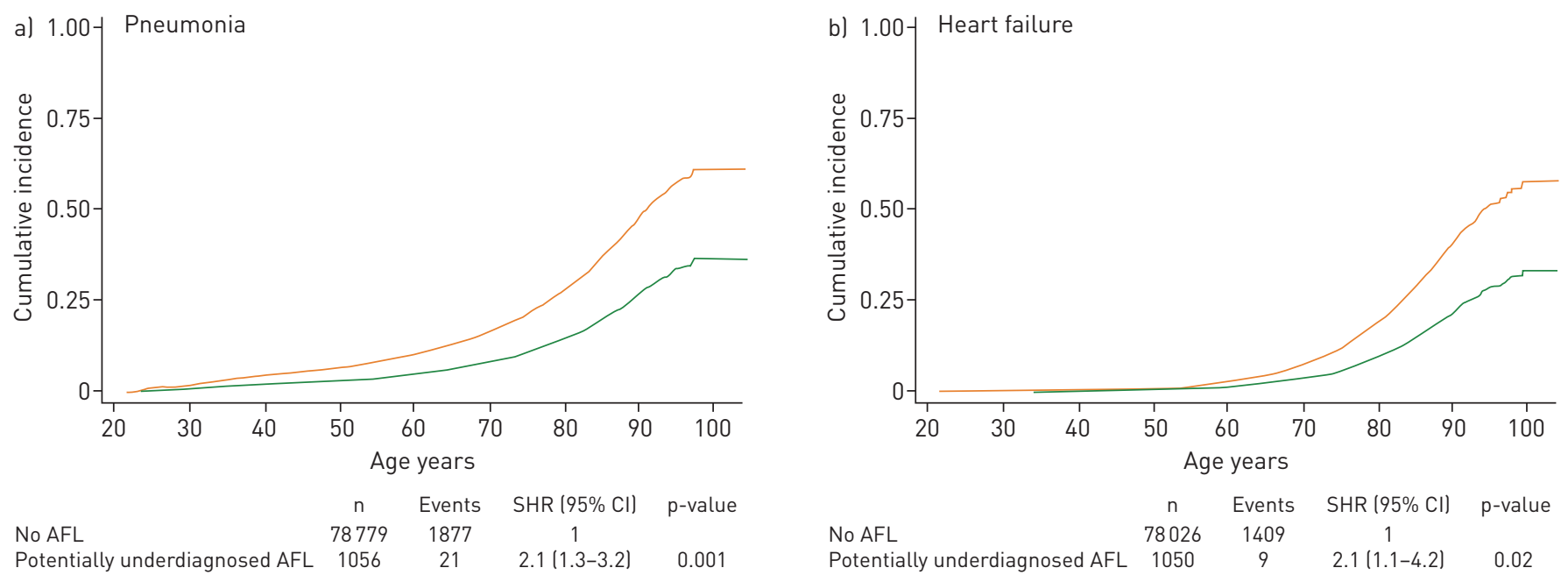

Potentially underdiagnosed AFL $1056 \quad 21 \quad 2.1(1.3-3.2) \quad 0.001$

$\begin{array}{lcccc} & \mathrm{n} & \text { Events } & \mathrm{SHR}(95 \% \mathrm{CI}) & \mathrm{p} \text {-value } \\ \text { No AFL } & 78026 & 1409 & 1 & \\ \text { Potentially underdiagnosed AFL } & 1050 & 9 & 2.1(1.1-4.2) & 0.02\end{array}$

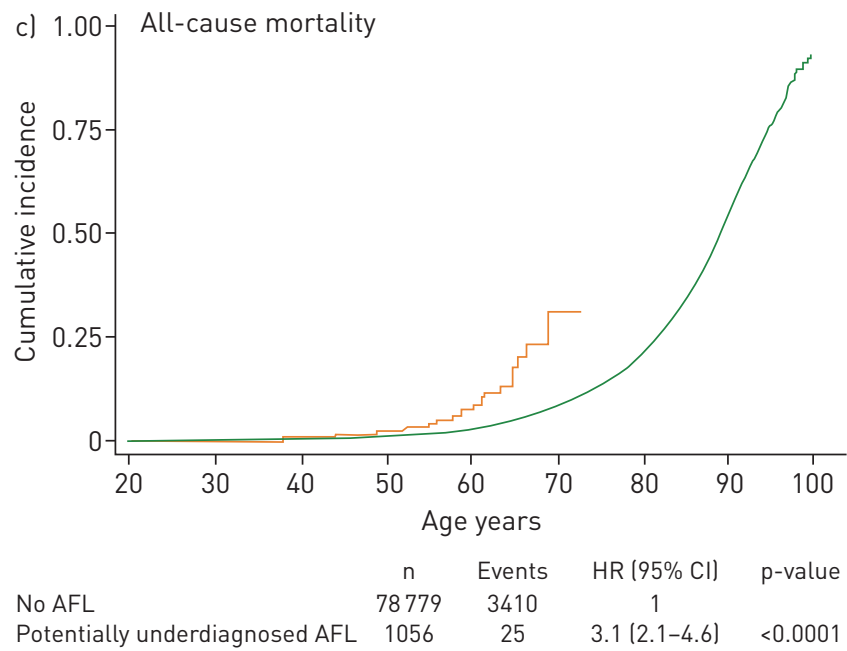

No AFL

Potentially underdiagnosed AFL

FIGURE 4 Competing risk analyses for pneumonia (a) and heart failure (b), and Kaplan-Meier analysis for all-cause mortality (c) in individuals with potentially underdiagnosed airflow limitation (AFL). All risk estimates were age- and sex-adjusted. Risk estimates for pneumonia and heart failure were acquired from competing risk analyses using the methods proposed by Fine and Gray with the competing event being all-cause mortality. Risk estimates for all-cause mortality were acquired from Cox proportional hazard models. It should be noted that risk for pneumonia was assessed using single failure-time analysis and not multiple failure-time analysis. p-Values were obtained from Wald tests. No AFL was defined as forced expiratory volume in $1 \mathrm{~s}\left(\mathrm{FEV}_{1}\right) /$ forced vital capacity (FVC) $\geqslant 0.70$ and $\mathrm{FEV} 1 / \mathrm{FVC} \geqslant$ lower limit of normal (LLN). Potentially underdiagnosed $\mathrm{AFL}$ was defined as FEV1/FVC $\geqslant 0.70$ and FEV1/FVC <LLN. Cl: confidence interval; HR: hazard ratio; SHR: subhazard ratio.

Alternatively, individuals with potentially underdiagnosed AFL may be a mixed clinical group consisting not only of those with respiratory disease, or the predisposition to develop it, but also those with heart disease, as these individuals displayed an increased risk of heart failure during follow-up compared to individuals without AFL. Heart failure has long been associated with AFL and it can often be a diagnostic challenge among patients with respiratory disease [16-18]. Thus, by using LLN among young individuals, we may perhaps not only identify those with increased risk of future respiratory disease but also future heart disease. Furthermore, among individuals experiencing an event with pneumonia, heart failure and/or death during follow-up, the Venn diagram only showed relatively limited overlap, which clearly indicates heterogeneity in outcome and suggests that different disease entities may be at play as the cause of poor prognosis.

Previous studies have focused mainly on older individuals with potentially overdiagnosed AFL and not on younger individuals with potentially underdiagnosed AFL. Overall, individuals with AFL according to the LLN but not the fixed ratio seem to be a group with an increased risk of future disease [19-22]. In the European Community Respiratory Health Survey, these individuals had an increased risk of developing \% predicted $\mathrm{FEV}_{1}<80 \%$ and of hospital service utilisation due to breathing problems after 9 years of follow-up [20]. In the third National Health and Nutrition Examination Survey, the same clinical group had the highest risk estimate (4.0) with regard to risk of all-cause mortality during up to 18 years of follow-up; however, the group only consisted of 20 individuals and statistical significance was not achieved 
FIGURE 5 Area-proportional Venn diagrams of pneumonia, heart failure, and all-cause mortality in individuals with potentially underdiagnosed airflow limitation (AFL). Potentially underdiagnosed AFL was defined as forced expiratory volume in $1 \mathrm{~s}$ (FEV 1 )/ forced vital capacity (FVC) $\geqslant 0.70$ and $F E V_{1} / F V C$ <lower limit of normal (LLN).

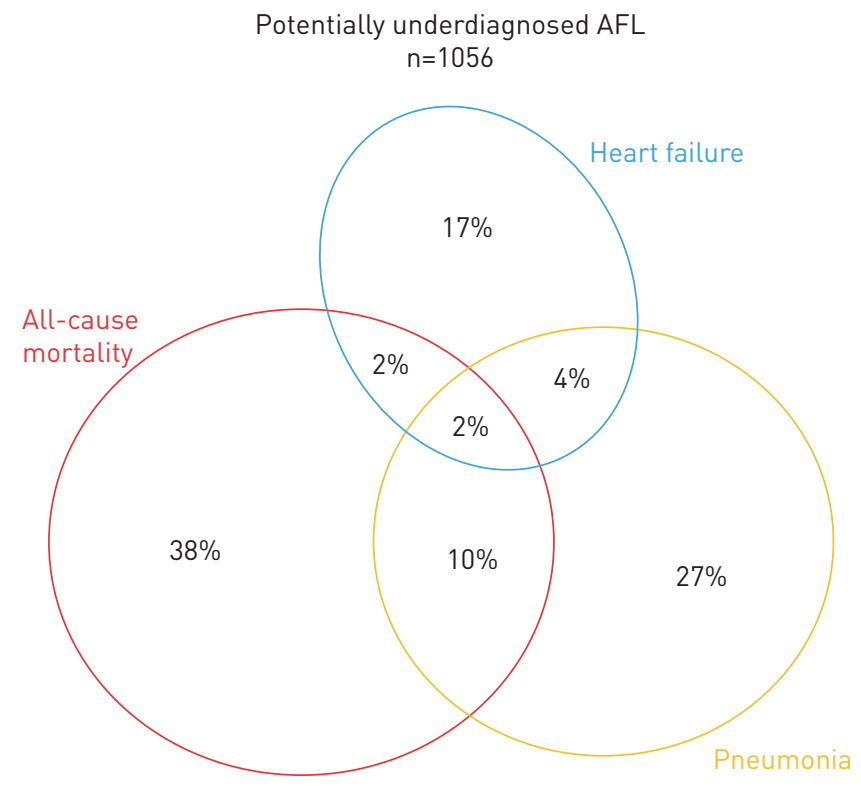

[21]. In the Lung Health Study, there was no overall difference in annual exacerbation rate compared to those without AFL over a 5-year period of annual follow-up; however, in the last year of follow-up, a noticeable increase could be observed, perhaps indicating development or progression of early COPD [19]. Furthermore, in the same cohort, there was no difference in decline of lung function [19], suggesting an absence of the accelerated lung function decline which usually characterises COPD [23].

In previous studies, individuals with AFL according to the fixed ratio but not the LLN have mostly been observed to have an increased risk of COPD-related exacerbations and all-cause mortality [19, 21, 24, 25]. In the present study, an increased risk of exacerbations of obstructive lung disease but not of all-cause mortality was found. These individuals seem to have clinical findings compatible with COPD [19, 25-28] and using the fixed ratio compared to the LLN among older individuals also seems to correlate better with the clinical diagnosis and prognosis of COPD [29]. Thus, based on previous studies and taken together with the present findings, it seems likely that these individuals may have undiagnosed COPD.

The strengths of the present study include a large sample selected randomly from the general population with information on spirometry and a long and complete follow-up time with clinically relevant outcomes. Furthermore, the study has a large number of individuals with discrepant definitions of AFL according to the LLN and the fixed ratio.

A major limitation of the study is the lack of post-bronchodilator measurements, which prevent us from classifying the type of AFL. Thus, some may have a reversible form of AFL (indicating asthma) and some may have an irreversible form of AFL (indicating COPD or asthma-COPD overlap). However, it is very clear that individuals with potentially underdiagnosed and overdiagnosed AFL have an increased risk of respiratory outcomes and possible misclassification due to lack of post-bronchodilator measurements would only tend to attenuate our findings rather than exaggerate them. Another limitation is that the results may not be transferable to other ethnicities besides those of white European ancestry.

In conclusion, defining AFL according to the LLN instead of the fixed ratio at younger ages seems to identify individuals with an increased risk of respiratory and cardiovascular morbidity and early death. Clinicians regularly performing spirometry in younger individuals should apply the LLN for the diagnosis of AFL, assuming the focus is on identifying a vulnerable population with subsequent high morbidity and mortality.

Conflict of interest: Y. Çolak has received personal fees from Boehringer Ingelheim and AstraZeneca outside the submitted work. J. Vestbo has received personal fees for consultancy for COPD phase 2 and 3 programmes from GlaxoSmithKline, Chiesi pharmaceuticals, Boehringer-Ingelheim, Novartis, Almirall and AstraZeneca, personal fees for consultancy for MRI software development from Bioxydyn, personal fees for lectures including service in speakers bureaus from GlaxoSmithKline, Chiesi pharmaceuticals, Novartis, AstraZeneca and Boehringer-Ingelheim, and personal fees from GlaxoSmithKline, AstraZeneca, Ferring, outside the submitted work. P. Lange has received grants and personal fees from Almirall, Boehringer Ingelheim and GSK, and personal fees from Astra Zeneca, Novartis, Nycomed, Pfizer and Mundipharma, outside the submitted work.

Support statement: The Lundbeck Foundation and the Danish Lung Association supported this work. The funders had no role in the design and conduct of the study, the collection, management, analysis or interpretation of the data, the 
preparation, review or approval of the manuscript, or the decision to submit the manuscript for publication. Funding information for this article has been deposited with the Crossref Funder Registry.

Author contributions: Y. Colak and P. Lange had full access to all of the data in the study and had final responsibility for the decision to submit for publication. Y. Çolak, S. Afzal, B.G. Nordestgaard, J. Vestbo and P. Lange contributed to the study concept and design. Y. Çolak, S. Afzal, B.G. Nordestgaard, J. Vestbo and P. Lange collected, analysed or interpreted the data. Y. Çolak wrote the draft manuscript. Y. Çolak, S. Afzal, B.G. Nordestgaard, J. Vestbo and P. Lange revised the manuscript for important intellectual content. Y. Colak performed the statistical analyses. B.G. Nordestgaard and P. Lange obtained the funding. B.G. Nordestgaard provided administrative, technical or material support. P. Lange supervised the study.

\section{References}

1 Çolak Y, Afzal S, Nordestgaard BG, et al. Prognosis of asymptomatic and symptomatic, undiagnosed COPD in the general population in Denmark: a prospective cohort study. Lancet Respir Med 2017; 5: 426-434.

2 National Institute for Health and Care Excellence. Chronic obstructive pulmonary disease in over 16s: diagnosis and management (CG101). www.nice.org.uk/guidance/cg101 Date last updated: June 2010. Date last accessed: October 20, 2017.

3 Vogelmeier CF, Criner GJ, Martinez FJ, et al. Global strategy for the diagnosis, management, and prevention of chronic obstructive lung disease 2017 report: GOLD executive summary. Eur Respir J 2017; 49: 1700214.

4 Guirguis-Blake JM, Senger CA, Webber EM, et al. Screening for chronic obstructive pulmonary disease: evidence report and systematic review for the US preventive services task force. JAMA 2016; 315: 1378-1393.

5 Mohamed Hoesein FA, Zanen P, Lammers JW. Lower limit of normal or FEV1/FVC $<0.70$ in diagnosing COPD: an evidence-based review. Respir Med 2011; 105: 907-915.

6 Çolak Y, Løkke A, Marott JL, et al. Impact of diagnostic criteria on the prevalence of COPD. Clin Respir J 2013; 7: 297-303.

7 Bakke PS, Rönmark E, Eagan T, et al. Recommendations for epidemiological studies on COPD. Eur Respir J 2011; 38: 1261-1277.

8 Pellegrino R, Viegi G, Brusasco V, et al. Interpretative strategies for lung function tests. Eur Respir J 2005; 26: 948-968.

9 Løkke A, Marott JL, Mortensen J, et al. New Danish reference values for spirometry. Clin Respir J 2013; 7: $153-167$.

10 Micallef L, Rodgers P. eulerAPE: drawing area-proportional 3-Venn diagrams using ellipses. PLoS One 2014; 9: e101717.

11 Postma DS, Bush A, van den Berge M. Risk factors and early origins of chronic obstructive pulmonary disease. Lancet 2015; 385: 899-909.

12 Agustí A, Noell G, Brugada J, et al. Lung function in early adulthood and health in later life: a transgenerational cohort analysis. Lancet Respir Med 2017; 5: 935-945.

13 Rennard SI, Drummond MB. Early chronic obstructive pulmonary disease: definition, assessment, and prevention. Lancet 2015; 385: 1778-1788.

14 Martinez FD. Early-life origins of chronic obstructive pulmonary disease. N Engl J Med 2016; 375: 871-878.

15 Lange P, Celli B, Agusti A, et al. Lung-function trajectories leading to chronic obstructive pulmonary disease. N Engl J Med 2015; 373: 111-122.

16 Brenner S, Güder G, Berliner D, et al. Airway obstruction in systolic heart failure-COPD or congestion? Int J Cardiol 2013; 168: 1910-1916.

17 Wannamethee SG, Shaper AG, Papacosta O, et al. Lung function and airway obstruction: associations with circulating markers of cardiac function and incident heart failure in older men-the British Regional Heart Study. Thorax 2016; 71: 526-534.

18 Lam CS, Lyass A, Kraigher-Krainer E, et al. Cardiac dysfunction and noncardiac dysfunction as precursors of heart failure with reduced and preserved ejection fraction in the community. Circulation 2011; 124: 24-30.

19 Akkermans RP, Biermans M, Robberts B, et al. COPD prognosis in relation to diagnostic criteria for airflow obstruction in smokers. Eur Respir J 2014; 43: 54-63.

20 Cerveri I, Corsico AG, Accordini S, et al. Underestimation of airflow obstruction among young adults using FEV1/FVC $<70 \%$ as a fixed cut-off: a longitudinal evaluation of clinical and functional outcomes. Thorax 2008; 63: $1040-1045$

21 Mannino DM, Diaz-Guzman E. Interpreting lung function data using $80 \%$ predicted and fixed thresholds identifies patients at increased risk of mortality. Chest 2012; 141: 73-80.

22 Pothirat C, Chaiwong W, Phetsuk N, et al. Misidentification of airflow obstruction: prevalence and clinical significance in an epidemiological study. Int J Chron Obstruct Pulmon Dis 2015; 10: 535-540.

23 Vestbo J, Edwards LD, Scanlon PD, et al. Changes in forced expiratory volume in 1 second over time in COPD. N Engl J Med 2011; 365: 1184-1192.

24 Mannino DM, Sonia Buist A, Vollmer WM. Chronic obstructive pulmonary disease in the older adult: what defines abnormal lung function? Thorax 2007; 62: 237-241.

25 Zaigham S, Wollmer P, Engstrom G. Lung function, forced expiratory volume in $1 \mathrm{~s}$ decline and COPD hospitalisations over 44 years of follow-up. Eur Respir J 2016; 47: 742-750.

26 Akkermans RP, Berrevoets MA, Smeele IJ, et al. Lung function decline in relation to diagnostic criteria for airflow obstruction in respiratory symptomatic subjects. BMC Pulm Med 2012; 12: 12.

27 Bhatt SP, Sieren JC, Dransfield MT, et al. Comparison of spirometric thresholds in diagnosing smoking-related airflow obstruction. Thorax 2014; 69: 409-414.

28 Mohamed Hoesein FA, Zanen P, Sachs AP, et al. Spirometric thresholds for diagnosing COPD: 0.70 or LLN, preor post-dilator values? COPD 2012; 9: 338-343.

29 Güder G, Brenner S, Angermann CE, et al. "GOLD or lower limit of normal definition? A comparison with expert-based diagnosis of chronic obstructive pulmonary disease in a prospective cohort-study". Respir Res 2012; 13: 13 . 